\title{
Microstructure, Corrosion Behaviors in Different Simulated Body Fluids and Cytotoxicity of Zn-Li Alloy as Biodegradable Material
}

\author{
Yu Zhang ${ }^{1,2}$, Yujiao $\mathrm{Lu}^{2,3}$, Xuemei $\mathrm{Xu}^{2}$, Liangjian $\mathrm{Chen}^{3}$, Tao $\mathrm{Xiao}^{4}$, Xier $\mathrm{Luo}^{2}$, \\ Yang $\mathrm{Yan}^{2}$, Ding $\mathrm{Li}^{4}$, Yilong $\mathrm{Dai}^{2}$ and $\mathrm{Kun} \mathrm{Yu}^{1,2, *}$ \\ ${ }^{1}$ Department of Materials Science and Engineering, Yantai Nanshan University, Yantai 265713, China \\ ${ }^{2}$ School of Materials Science and Engineering, Central South University, Changsha 410083, China \\ ${ }^{3}$ The Third Xiangya Hospital, Central South University, Changsha 410013, China \\ ${ }^{4}$ The Second Xiangya Hospital, Central South University, Changsha 410011, China
}

Zn-based biodegradable materials have aroused wide attention due to promising biodegradability and adaptability to tissue regeneration in recent years. $\mathrm{Zn}-0.5 \% \mathrm{Li}$ alloys was cast and hot extruded in this study. The microstructure, electrochemical properties and cytocompatibility of the as-extruded $\mathrm{Zn}-0.5 \% \mathrm{Li}$ alloy were investigated. Four different simulated body fluids (Ringer's solution, simulated body fluid (SBF), Dulbecco's Modified Eagle Medium (DMEM) and DMEM-supplementing with $10 \%$ fetal bovine serum (DMEMp)) were employed to study the corrosion behavior of the alloy. The result indicated that $\mathrm{Zn}-0.5 \% \mathrm{Li}$ alloy was composed of the matrix $\mathrm{Zn}$ and the second phase $\mathrm{LiZn}$. It showed a higher corrosion resistance in the buffer solutions than that in Ringer's solution, the corrosion rate can be ranked as Ringer's $>$ DMEMp $>$ DMEM $>$ SBF. What's more, the cytotoxicity test shows that the alloy is not toxic to BMSCs. Zn-0.5\%Li alloy has the potential to be the biodegradable metal. [doi:10.2320/matertrans.M2018342]

(Received October 29, 2018; Accepted December 26, 2018; Published March 25, 2019)

Keywords: Zn-Li alloy, microstructure, degradation behavior, simulated body fluids, biocompatibility

\section{Introduction}

$\mathrm{Zn}$ alloys is a new biodegradable metals (BMs) developed on the basis of the Mg-based and Fe-based BMs materials. Compared with biomedical materials used in clinic, it has the following advantages. $\mathrm{Zn}$ shows a good compatibility. $\mathrm{Zn}$ is one of the necessary elements in human body, which is widely involved in the metabolism process of the body and is essential for the bone formation. $\mathrm{Zn}$ has an appropriate degradable rate. The standard potential of pure $\mathrm{Zn}$ $(-0.763 \mathrm{~V})$ is between that of pure $\mathrm{Mg}(-2.37 \mathrm{~V})$ and that of pure $\mathrm{Fe}(-0.44 \mathrm{~V})$, which resulted in an optimal degradable rate $(10-20 \mu \mathrm{m} / \mathrm{yr})$ when $\mathrm{Zn}$ was used as a biodegradable metallic stent. ${ }^{1)} \mathrm{Zn}$ alloys exhibit suitable mechanical properties. Although pure $\mathrm{Zn}$ shows poor properties in both strength and hardness that can't meet the requirement of biomaterials implantation. ${ }^{2)}$ Adding alloying elements and thermo-mechanical refinement could effectively improve the mechanical properties of pure Zn. Among Zn-X $(\mathrm{Mg}, \mathrm{Ca}, \mathrm{Cu}, \mathrm{Li}$ et al.) alloy systems, $\mathrm{Zn}-\mathrm{Li}$ alloy exhibited relatively remarkable comprehensive properties including excellent mechanical properties, promise biodegradable rate and good biocompatibility. ${ }^{1,3-5)}$ Thus $\mathrm{Zn}-\mathrm{Li}$ alloy is considered as the next promising generation of $\mathrm{BMs}$ for ideal biodegradable implants.

In vitro degradation behaviors of biodegradable metals vary in different degradation environments including solution types, surface area to volume ratio, $\mathrm{pH}$ value and temperature. The presence of different kinds of compounds can change the solubility of zinc dissolved products. Zinc complexes formed on the surface can increase the conductivity of electrolytes, whilst change the composition, structure and density of corrosion products. ${ }^{6}$ What's more, the anions in different immersion solutions have great impact

*Corresponding author, E-mail: yukun2010@csu.edu.cn on the corrosion process to a large extent. ${ }^{7,8)}$ What effect the anions in human body have on the corrosion behavior of $\mathrm{Zn}$ alloy has not been clear yet. Therefore it's necessary to study the corrosion behaviors of $\mathrm{Zn}$ alloy in different simulated body fluids, and observe the morphology characteristics of corrosion products.

\section{Materials and Methods}

$\mathrm{Zn}-0.5 \% \mathrm{Li}$ alloy was prepared by melting high purity $\mathrm{Zn}$ (99.99\%), Li (99.999\%) in a resistance furnace at $550^{\circ} \mathrm{C}$ under an argon atmosphere. The molten metal was then poured into a preheated cylindrical steel mold to achieve the ingot $(\varnothing 60 \mathrm{~mm} \times 200 \mathrm{~mm})$. Subsequently, annealing at $300^{\circ} \mathrm{C}$ for $48 \mathrm{~h}$ to homogenize the cast microstructure and then quenching in water. The ingot was hot extruded to obtain the plates of $4 \mathrm{~mm}$ in thickness at $250^{\circ} \mathrm{C}$ with the extrusion ratio of 16:1. The specimens for microstructure characteristic and in vitro corrosion tests were cut into plates with the size of $10 \mathrm{~mm} \times 10 \mathrm{~mm} \times 4 \mathrm{~mm}$.

Sample for microscopic observation was polished, etched with acid solution and observed with optical microscopy. The DMAX-2500X X-ray diffractometery (XRD) was employed for identification of constituent phases with the values of $10^{\circ}-90^{\circ}$. Specimens for TEM observation came from the plate, mechanically thinned to $80 \mu \mathrm{m}$ and then reduced by electrolytic jet polishing in a solution of $10 \% \mathrm{HClO}_{4}+90 \%$ $\mathrm{C}_{2} \mathrm{H}_{5} \mathrm{OH}$ at $20 \mathrm{~V}$ between $-30^{\circ} \mathrm{C}$ and $-20^{\circ} \mathrm{C}$.

The immersion tests were carried out in solutions at $37 \pm$ $0.2^{\circ} \mathrm{C}$ and $5 \% \mathrm{CO}_{2}$ atmosphere for 30 days to detect the $\mathrm{pH}$ value and weight change. The ratio of the solution volume to sample surface area was $20 \mathrm{~cm}^{3} / \mathrm{cm}^{2}{ }^{10}$ ) Four primary solutions were used in immersion tests and potentiodynamic polarization tests: Ringer's solution, simulated body fluid (SBF), Dulbecco's Modified Eagle Medium (DMEM) and DMEM-supplementing with $10 \%$ fetal bovine serum 

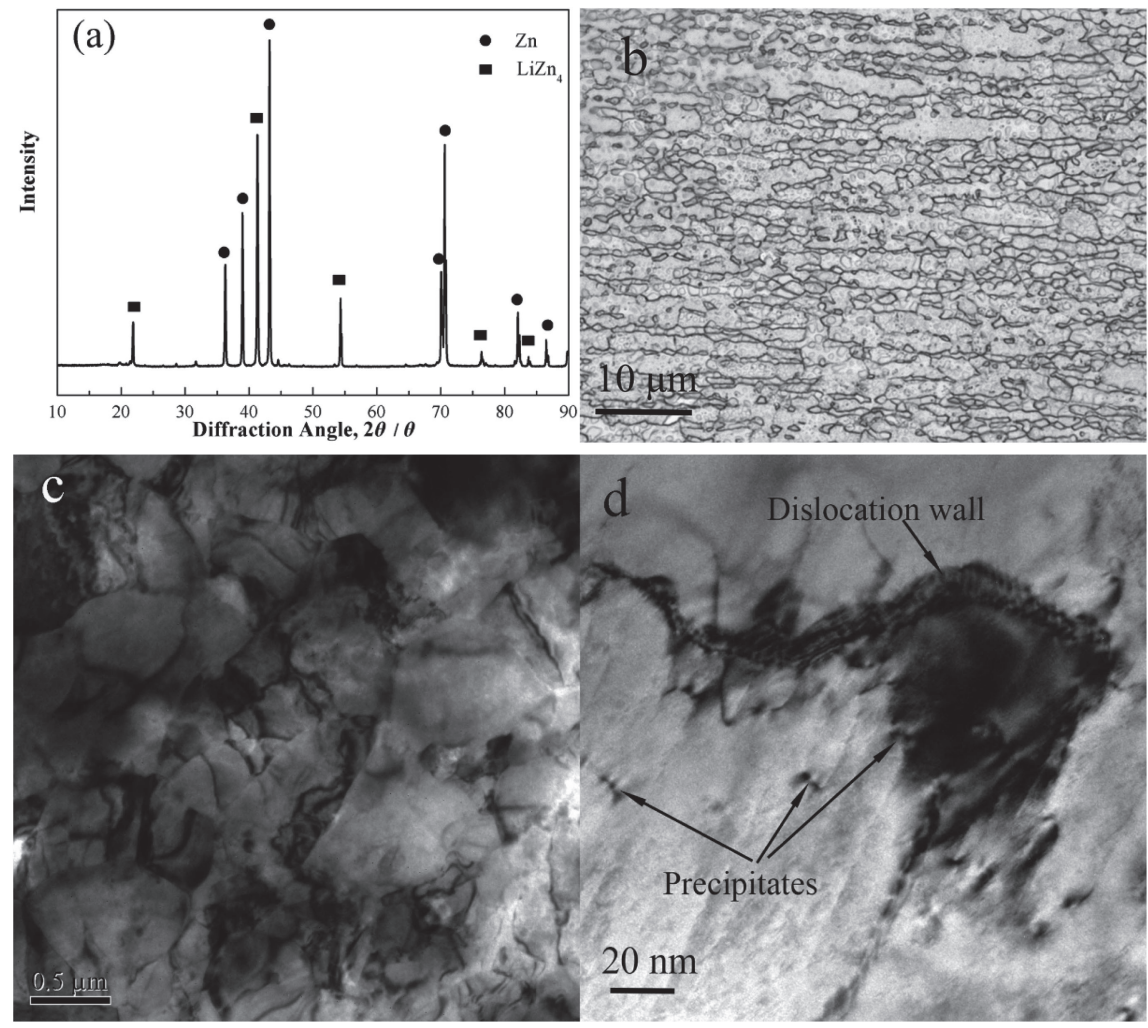

Fig. 1 (a) X-ray diffraction pattern, (b) optical micrograph in the extrusion direction and (c, d) TEM bright-field micrographs of as-extruded $\mathrm{Zn}-0.5 \% \mathrm{Li}$ alloy.

(DMEMp). The $\mathrm{pH}$ value of solutions was adjusted to 7.4 before testing. Tafel polarization curves were determined at $37^{\circ} \mathrm{C}$ using an electrochemical workstation (CHI660C) by a standard three-electrode configuration: saturated calomel as a reference electrode, platinum mesh as the counter and the sample as a working electrode. The specimens were polished with 1000 grit $\mathrm{SiC}$ emery papers and sealed with epoxy resin with an exposed surface of $1 \mathrm{~cm}^{2}$. The tested surfaces were taken from the ED-TD sections (surface parallel to the extruded face). The potentiodynamic polarization curve was measured at a scan rate of $1 \mathrm{mV} / \mathrm{s}$ with a potential range of $-1.8 \sim-0.8 \mathrm{~V}$. According to ASTM-G102-89, the corrosion rate $(\mathrm{CR})$ can be calculated by extrapolating the polarization curve. ${ }^{11)}$ Surface corrosion morphologies were observed by SEM (Quanta 200) with EDS.

Bone marrow mesenchymal stem cells (BMSCs) were selected to carry out the cytotoxicity test. The cytotoxicity test was conducted using indirect contact method. The extract was taken from the sample immersing in the DMEMp for $72 \mathrm{~h}$ at a humidified atmosphere containing $5 \% \quad \mathrm{CO}_{2}$ at $37^{\circ} \mathrm{C}$ according to ISO 10993-5: 1999. The extract was diluted to $50 \%$ and $10 \%$ concentration respectively, and then refrigerated at $4^{\circ} \mathrm{C}$. Cells were seeded inside three 96-well culture plates with a cell density of $5 \times 10^{4}$ cells $/ \mathrm{cm}^{3}$ for each well and incubated in a humidified atmosphere containing $5 \% \quad \mathrm{CO}_{2}$ at $37^{\circ} \mathrm{C}$ for $24 \mathrm{~h}$. Then the medium was successively replaced with $100 \mu \mathrm{L}$ by $100 \%, 50 \%$ or $10 \%$ extracts, or a negative control (culture medium), or a positive control (containing $0.64 \%$ phenol). Then, $10 \mu \mathrm{L}$ CCK-8 solution was added to each well and further incubated for $4 \mathrm{~h}$. The absorbance spectra of supernatant were calculated using a microplate reader at $474 \mathrm{~nm}$.

\section{Results and Discussion}

Figure 1(a) shows the XRD pattern of as-extruded $\mathrm{Zn}$ $0.5 \% \mathrm{Li}$ alloy comprising of $\mathrm{Zn}$ matrix and LiZn4 second phase. $\mathrm{Zn}-0.5 \% \mathrm{Li}$ alloy is approximately eutectic according to the equilibrium phase diagram, the result is same as the previous research. ${ }^{12)}$ Figure 1(b) exhibits the optical metallographic microstructure of the alloy in extrusion direction. It shows a fibrous structure with the grain crushed, lengthened into fibroid and distributes along the extrusion direction. The microstructure is refined and the average size of grain is $4.3 \mu \mathrm{m}$, indicating that the material has undergone a dynamic recrystallization during the process of hot extrusion since $\mathrm{Zn}$ has a low dynamic recrystallization temperature. Figure 1(c) and Fig. 1(d) show the TEM bright-field micrographs of asextruded $\mathrm{Zn}-0.5 \% \mathrm{Li}$ alloy. Figure 1(c) shows a typical deformation microstructure that grains are stretched and fine subgrains appear in the area of the gain boundary intersection. In the Fig. 1(d) dislocation wall along the grain boundary is observed, a series of bean-like $\mathrm{LiZn}_{4}$ precipitates uniformly distribute in the interior of the grain. Since $\mathrm{Zn}$ matrix and precipitate $\mathrm{LiZn}_{4}$ have the same crystal structure (hcp), their lattice parameters are very close too, with $\mathrm{a}=0.2664$ and $\mathrm{a}=0.2788 \mathrm{~nm}$ respectively. ${ }^{13)}$ Consequently, the lattice mismatch between the $\mathrm{Zn}$ matrix and the $\mathrm{LiZn}_{4}$ precipitate is very low, which can decrease the nucleation barrier for precipitates. The difference of lattice parameter 

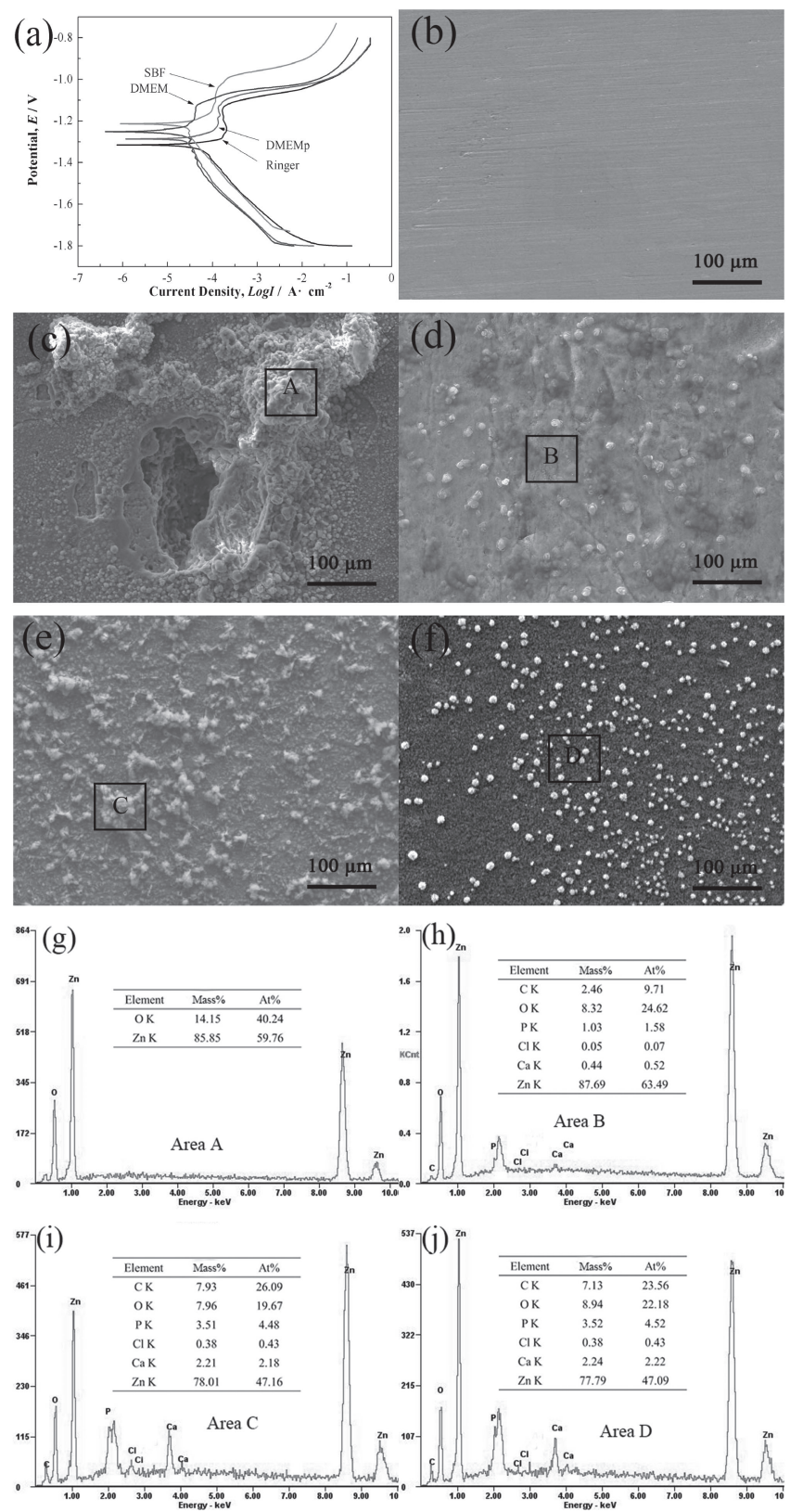

Fig. 2 (a) Typical potentiodynamic polarization curves, (b) surface morphology before the immersion test, $(c-j)$ surface morphologies and EDS results of corresponding corrosion products in Ringer's solution, SBF, DMEM and DMEMp respectively for 7 days.

between the substrate and the nucleation phase is smaller, the lattice mismatch would be better and the interface energy would be lower, the heterogeneous nucleation is more likely to happen.

Figure 2(a) shows the polarization curves of the asextruded $\mathrm{Zn}$-Li alloy in four different simulated body fluids. Table 1 summarizes the electrochemical parameters, corrosion rates calculated from electrochemical measurement $\left(\mathrm{CR}_{\text {coor }}\right)$. The corrosion potentials $\left(E_{\text {corr }}\right)$ of the $\mathrm{Zn}-\mathrm{Li}$ alloy are $-1.315 \mathrm{~V}$ in Ringer's solution, $-1.213 \mathrm{~V}$ in SBF, $-1.260 \mathrm{~V}$ in DMEM and $-1.286 \mathrm{~V}$ in DMEMp. The corrosion potential in Ringer's solution is higher than those in buffer solutions. Besides, the corrosion current density ( $I_{\text {corr }}$ ) and $C R_{\text {coor }}$ in Ringer's solution is improved compared with those in buffer solutions too, which may be involved in
Table 1 Electrochemical data and immersion test data of the alloy in different simulated body fluids.

\begin{tabular}{cccccc}
\hline Solution & $\begin{array}{c}E_{\text {corr }} \\
(\mathrm{V})\end{array}$ & $\begin{array}{c}I_{\text {corr }} \\
\left(\mu \mathrm{A} / \mathrm{cm}^{2}\right)\end{array}$ & $\begin{array}{c}C R_{\text {corr }} \\
(\mathrm{mm} / \mathrm{yr})\end{array}$ & $\begin{array}{c}C R_{\text {it }} \\
(\mathrm{mm} / \mathrm{yr})\end{array}$ & $\mathrm{pH}$ \\
\hline Ringer & -1.315 & 11.31 & 0.169 & 0.633 & 8.7 \\
SBF & -1.213 & 3.67 & 0.055 & 0.110 & 8.0 \\
DMEM & -1.260 & 6.24 & 0.093 & 0.137 & 8.0 \\
DMEMp & -1.286 & 8.37 & 0.125 & 0.219 & 8.0 \\
\hline
\end{tabular}

forming a protective passive layer on the surface then decreasing corrosion of the substrate. In the meanwhile, the anodic branches of four polarization curves show the same current platform, implying that $\mathrm{Zn}-\mathrm{Li}$ alloy has a high corrosion resistance as the formation of a protective passive layer on the surface. The length of platform could be used to measure the stability of passive layer. The longer the platform is, the passive layer is thicker and is more resistant to corrosion. And the break-down potential of the platform means the passive layer starts to being destroyed. The curve in the Ringer's solution exhibits the shortest platform and the most negative break-down potential among those solutions, implying alloy has the worst corrosion resistance in the Ringer's solution. On the contrary, the alloy has a better corrosion resistance in the buffer solutions. In addition the $\mathrm{CR}_{\text {coor }}$ can be ranked as follows: Ringer's $>$ DMEMp $>$ $\mathrm{DMEM}>\mathrm{SBF}$. The $\mathrm{pH}$ value in Ringer's solution is much higher than that in buffer solutions.

Table 1 also lists the corrosion rates $\left(\mathrm{CR}_{\mathrm{it}}\right)$ and $\mathrm{pH}$ value measured from the immersion test in the four different simulated body fluids for $30 \mathrm{~d}$. The $\mathrm{CR}_{\mathrm{it}}$ measured from the immersion test is greater than $\mathrm{CR}_{\text {coor }}$ calculated from the electrochemical measurement. The order of $\mathrm{CR}_{\mathrm{it}}$ is consistent with the result of $\mathrm{CR}_{\text {coor }}$. The $\mathrm{pH}$ value in Ringer's solution reaches at 8.7 for $30 \mathrm{~d}$, and the $\mathrm{pH}$ values in buffer solutions all keep at 8.0 eventually. A lower $\mathrm{pH}$ value reflects the higher corrosion resistance of the alloy since the generation of extra $\mathrm{OH}^{-}$would lead to the increase of $\mathrm{pH}$ value. According to the result, the alloy in buffer solution has a higher corrosion resistance than that in Ringer's solution.

Figure 2(c)-(f) show the surface morphology of the samples immersed in the four different simulated body fluids for $7 \mathrm{~d}$ and Fig. 2(g)-(j) show the EDS analysis of corresponding corrosion product. Compared with the surface morphology (Fig. 2(b)) of specimen before the immersion test. The alloy in Ringer's solution exhibits the characteristic of localized corrosion, the accumulation of voluminous corrosion products and the deep corrosion pit, which may be involved in the micro-galvanic corrosion between the substrate and the second phase. The alloy in the SBF shows the characteristic of uniform corrosion with the formation of passive layer, the spherical corrosion products on the passive layer are smaller than those in Ringer's solution. In case of the alloy in DMEM the passive layer is more denser, the corrosion is uniform and the number of cotton-like corrosion products is larger. For the alloy in DMEMp, the white spherical corrosion products uniformly distribute on the passive layer. The EDS results show the corrosion product in Ringer's solution after $7 \mathrm{~d}$ mainly consists of $\mathrm{Zn}$ and $\mathrm{O}$. It can be assumed that the corrosion product is zinc oxide. In 

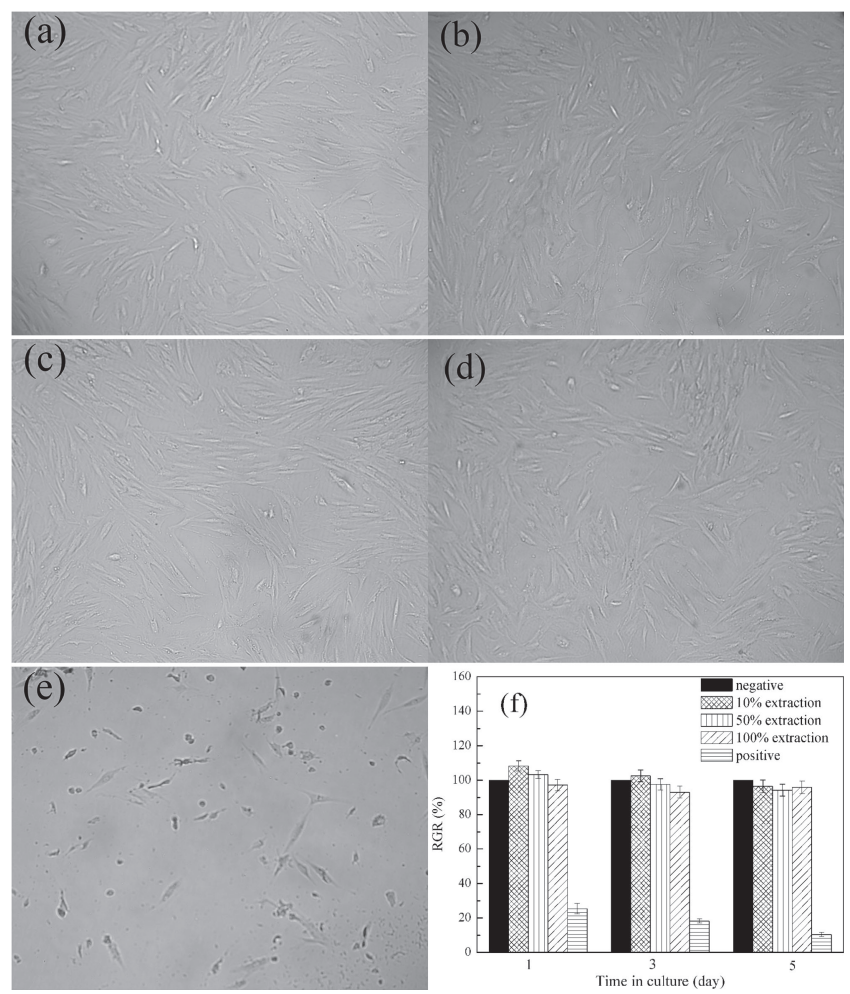

Fig. 3 BMSCs morphology in 5 days incubation: (a) negative control; (b) $10 \%$ extraction; (c) $50 \%$ extraction; (d) $100 \%$ extraction of the alloy; (e) positive control; (f) RGR of BMSCs in 1, 3 and 5 days incubation in $10 \%, 50 \%$ and $100 \%$ extracts of the alloy.

addition, more $\mathrm{C}, \mathrm{P}, \mathrm{Ca}$ are detected in the corrosion products in buffer solutions, assuming that corrosion products mainly contain more carbonates, phosphates. The results are in accordance with the corrosion products reported in the literature. ${ }^{11)}$ As can be seen in above results, the corrosion rates in SBF, DMEM and DMEMp containing $\mathrm{HCO}_{3}{ }^{-}$and $\mathrm{HPO}_{4}{ }^{2-}$ are lower than that in Ringer's solution. The change of corrosion behavior might due to the types of ions in fluids, suggesting that the corrosion behavior of the alloy is mainly influenced by the solution type. $\mathrm{Cl}^{-}$plays a negative effect on the corrosion of the alloy as it destroys the passive layer, which is similar to the effect of $\mathrm{Cl}^{-}$on $\mathrm{Mg}$ alloy. While $\mathrm{HCO}_{3}{ }^{-}$and $\mathrm{HPO}_{4}{ }^{2-}$ play the positive effect on the corrosion of alloy owing to the formation of a protective zinc phosphate film. There was a similar phenomenon in $\mathrm{Mg}$ alloys, the corrosion decreased when the presence of bicarbonate in solution that may improve the formation of a protective layer on the surface. ${ }^{13)}$ The protein is responsible for the increased corrosion rate in this experiment.

Figure 3(a)-(e) shows the morphologies of BMSCs for 5 days of incubation in different extract mediums with concentrations of 10, 50 and $100 \%$. The BMSCs morphologies in different extract mediums are in health and flattened spindle shape, similar to that in the negative control. Figure 3(f) shows the relative growth rate (RGR) of BMSCs after 1, 3 and 5 days of incubation in the extract medium, respectively. The result shows no statistical differences detected in the RGR of the different extract mediums and the negative control $(p>0.05)$, while there is a significant difference between the extract groups and positive group
( $p<0.05$ ). According to ISO 10993-5: 1999, the cytotoxicity of these extracts of the alloy is in Grade $0-1$. In other words, the as-extruded $\mathrm{Zn}-\mathrm{Li}$ alloy is innocuous and satisfactory for biodegradable implant material.

\section{Conclusion}

The microstructure observations demonstrated that the microstructure of $\mathrm{Zn}-0.5 \% \mathrm{Li}$ alloy is uniform and the grain is refined, the alloy consists of the matrix $\mathrm{Zn}$ and the nanoprecipitate $\mathrm{LiZn}_{4}$. The electrochemical measurements and immersion tests showed that the alloy has a low corrosion rate $(\leq 0.633 \mathrm{~mm} / \mathrm{yr})$, besides the corrosion resistance of the alloy in the buffer solutions is much higher than that in Ringer's solution. The corrosion products analysis indicated that the majority of corrosion product is zinc oxide and a small part of that are carbonates and phosphates. Which suggests $\mathrm{Cl}^{-}$accelerates the corrosion rate of the alloy, $\mathrm{HCO}^{3-}$ and $\mathrm{HPO}_{4}{ }^{2-}$ retard the corrosion of alloy by forming a protective passive film. Moreover, the cytotoxicity results showed that the alloy is not toxic to BMSCs. As a result, $\mathrm{Zn}-0.5 \% \mathrm{Li}$ alloy can be regarded as the potential biodegradable metal.

\section{Acknowledgements}

The authors acknowledge of the 2015 ShanDong province project of outstanding subject talent group, the Natural Science Foundation of ShanDong Province of China (ZR2017MEM005), the project (2017GK2120) supported by the Key Research and Development Program of Hunan Province and the Natural Science Foundation of Hunan Province of China (2018JJ2506).

\section{REFERENCES}

1) P.K. Bowen, G.R. Nd, E.R. Shearier, J.M. Seitz, J. Drelich, M. Bocks, F. Zhao and J. Goldman: Mater. Sci. Eng. C 56 (2015) 467-472.

2) P.K. Bowen, E.R. Shearier, S. Zhao, R.J. Guillory, F. Zhao, J. Goldman and J.W. Drelich: Adv. Healthc. Mater. 5 (2016) 1121-1140.

3) S. Zhao, J. Seitz, R. Eifler, H.J. Maier, R.J. Guillory, E.J. Earley, A. Drelich, J. Goldman and J.W. Drelich: Mater. Sci. Eng. C 76 (2017) 301-312.

4) C. Yao, Z. Wang, S.L. Tay, T. Zhu and W. Gao: J. Alloys Compd. 602 (2014) 101-107.

5) Z. Tang, J. Niu, H. Huang, H. Zhang, J. Pei, J. Ou and G. Yuan: J. Mech. Behav. Biomed. 72 (2017) 182.

6) X.G. Zhang: Corrosion and Electrochemistry of Zinc, (Springer Science+Business Media, New York, 1996) pp. 28-29.

7) T. Ashino, N. Ohtsu and K. Wagatsuma: Mater. Trans. 49 (2008) $1342-$ 1345 .

8) D. Kuroda, S. Hiromoto, T. Hanawa and Y. Katada: Mater. Trans. 43 (2002) 3100-3104

9) I.A.B.O. American Society for Testing Materials, Philadelphia, PA, (2004).

10) ASTM G102-89, Standard, Practice for Calculation of Corrosion Rates and Related Information from Electrochemical Measurements, (2004).

11) S. Zhao, C.T. McNamara, P.K. Bowen, N. Verhun, J.P. Braykovich, J. Goldman and J.W. Drelich: Metall. Mater. Trans. A 48 (2017) 12041215.

12) A.D. Pelton: J. Phase Equilib. 12 (1991) 42-45.

13) J.F. Ferguson and P.L. Mccarty: Environ. Sci. Technol. 5 (1971) 534 540 . 